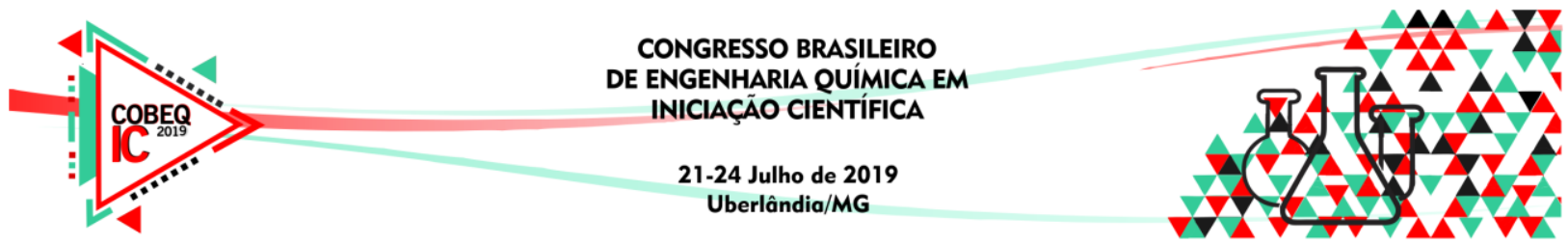

\title{
ESTUDO NUMÉRICO DO EFEITO DAS PROPRIEDADES DAS PARTÍCULAS E DA VELOCIDADE DE IMPACTO SOBRE O COEFICIENTE DE RESTITUIÇÃO
}

\author{
K. R. B. MELO' ${ }^{1}$, J. P. F. CAMPOS ${ }^{1}$ e G. C. LOPES $^{1}$ \\ ${ }^{1}$ Universidade Federal de São Carlos, Departamento de Engenharia Química \\ E-mail para contato: karlaraphaelamelo@gmail.com
}

\begin{abstract}
RESUMO - As colisões entre partícula e superfície desempenham um importante papel no comportamento fluidodinâmico e na dissipação de energia em escoamentos sólido-fluido. A melhor compreensão dessas colisões se dá através de estimativas de coeficiente de restituição. Na fluidodinâmica computacional, esse parâmetro é tido como essencial para os modelos de contato presentes em simulações que utilizam o Método dos Elementos Discretos (DEM). Sendo assim, para representar escoamentos sólido-fluido com precisão, é fundamental que os valores de entrada desse coeficiente sejam constantemente aprimorados. Nesse contexto, este trabalho teve como objetivo simular colisões entre partículas esféricas e superfície a fim de avaliar a influência das propriedades elásticas das partículas e da velocidade de impacto sobre o coeficiente de restituição. Para tanto, foram realizadas simulações utilizando o software de código aberto CFDEM ${ }^{\circledR}$ coupling. Os resultados apresentaram uma boa concordância quando comparados com os dados experimentais. Além disso, as simulações descreveram de modo satisfatório o significado físico do escoamento, indicando a diminuição do coeficiente de restituição para maiores velocidades de impacto e menor módulo de Young.
\end{abstract}

\section{INTRODUÇÃO}

Escoamentos sólido-fluido são amplamente utilizados em processos industriais, como em etapas de cristalização e sedimentação, operações hidrometalúrgicas, contra lavagem de filtros granulares, adsorção e troca iônica, tratamento de efluentes e outras aplicações. Nesses processos, as características mecânicas, físicas e elásticas das partículas da fase sólida, desempenham um importante papel no comportamento fluidodinâmico, sendo de grande relevância para o entendimento das interações físicas em escoamento sólido-fluido (Du, 2006). Essas interações ocorrem principalmente por meio de colisões entre as partículas ou entre as partículas e superfícies. A melhor compreensão dessas colisões dá-se através de estimativas de coeficiente de restituição $(e)$.

O coeficiente de restituição é expresso como a razão entre as velocidades relativas de dois objetos antes e após a colisão. A partir dele, pode-se mensurar a forma como parte da energia cinética das partículas é dissipada ao longo do escoamento. Os valores de $e$ variam entre 0 e 1 , sendo este igual a 0 em sistemas em que toda a energia é dissipada e 1 para colisões perfeitamente elásticas, em que há conservação da energia do sistema (Albuquerque, 


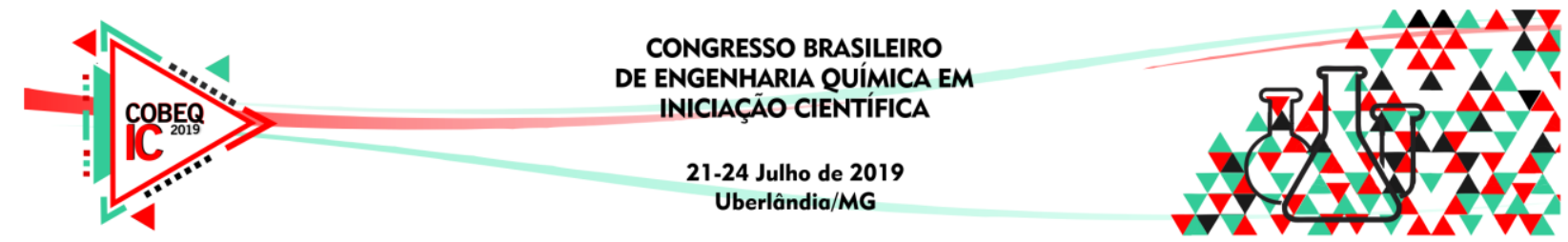

2016). Na literatura, os valores de $e$ são obtidos principalmente em experimentos para colisões de esferas rígidas e lisas. No entanto, esse parâmetro é variável e pode ser dependente de propriedades físicas e elásticas das partículas, das características da superfície de colisão, do fluido do escoamento e das velocidades de impacto (Gondret, 2002; Joseph et al., 2001).

\section{MODELAGEM MATEMÁTICA}

A metodologia experimental proposta por Lopes et al. (2018) foi reproduzida nas simulações numéricas, utilizando uma abordagem Euleriana-Lagrangeana, no software CFDEM $^{\circledR}$ coupling. Esse acopla dados obtidos para o fluido através do código OpenFOAM ${ }^{\circledR}$, aos dados obtidos para as partículas através do código LIGGGHTS ${ }^{\circledR}$. O acoplamento entre as fases fluido-partículas é feito através do cálculo da fração volumétrica de partículas que ocupam cada uma das células da malha computacional.

As variáveis de pressão e velocidade do fluido são calculadas pelo Método dos Volumes Finitos através das equações da continuidade e de Navier-Stokes implementadas no OpenFOAM $^{\circledR}$, as quais são resolvidas de acordo com as mudanças no movimento do fluido e o deslocamento das partículas discretas. A trajetória das partículas é determinada através do Método dos Elementos Discretos (DEM) pelo software LIGGGHTS ${ }^{\circledR}$.

O acoplamento CFD-DEM foi utilizado a fim de possibilitar simulações futuras com fluidos de maior viscosidade, como a água. Nas simulações realizadas, foi utilizado o modelo de contato partícula-partícula e partícula-superfície Hertz-Mindlin.

\subsection{Malha computacional}

A malha tridimensional utilizada nas simulações foi construída com a ferramenta blockMesh no OpenFOAM ${ }^{\circledR}$. Os dados referentes a ela são apresentados na Tabela 1.

Tabela 1 - Dados da malha

$\begin{array}{cc}\text { Tipo } & \text { Hexaédrica } \\ \text { Geometria } & \text { Tridimensional } \\ \text { Comprimento }(\mathbf{m}) & 0,5 \\ \text { Largura }(\mathbf{m}) & 0,2 \\ \text { Altura }(\mathbf{m}) & 1,5 \\ \text { Número de divisões em cada direção } \\ (\mathbf{x} \text { y z })\end{array}$

\subsection{Parâmetros das simulações}

Durante as simulações, foram registrados os dados de posição, velocidade linear e força a cada 150 passos de tempo. Para determinar essa condição, foi realizado um teste de independência do número de imagens/segundo. 


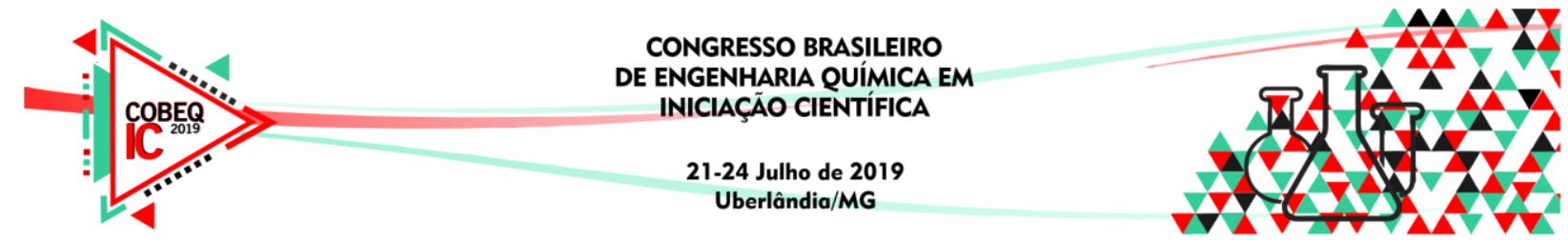

As propriedades das partículas esféricas e do fluido usados nas simulações estão dispostas na Tabela 2. O material considerado para a superfície contra a qual as partículas colidiram foi o vidro, com módulo de Young igual a $7 \times 10^{10} \mathrm{~Pa}$.

Tabela 2 - Propriedades físicas e elásticas das partículas

\begin{tabular}{cccccc}
\hline & & $\begin{array}{c}\text { Densidade } \\
\left(\mathrm{kg} / \mathrm{m}^{3}\right) \pm \sigma\end{array}$ & $\begin{array}{c}\text { Diâmetro }(\mathrm{mm}) \pm \\
\sigma\end{array}$ & $\begin{array}{c}\text { Módulo de } \\
\text { Young (Pa) }\end{array}$ & $\begin{array}{c}\text { Coeficiente de } \\
\text { restituição }\end{array}$ \\
\hline $\begin{array}{c}\text { Partículas } \\
\text { esféricas }\end{array}$ & $\begin{array}{c}\text { ABS com } \\
\text { recobrimento } \\
\text { uniforme }\end{array}$ & $1913 \pm 10$ & $5,96 \pm 0,01$ & $5,67 \times 10^{8}$ & 0,735 \\
\hline Fluido & Tapioca & $1080 \pm 6$ & $12,18 \pm 0,56$ & $3,27 \times 10^{5}$ & 0,405 \\
\hline
\end{tabular}

Fonte: Lopes et al., 2018

Os parâmetros das simulações encontram-se na Tabela 3.

Tabela 3 - Parâmetros das simulações

\begin{tabular}{cc}
\hline Escoamento & Laminar \\
Tempo de simulação & $1 \mathrm{~s}$ \\
Passo de tempo LIGGGHTS & $10^{-5} \mathrm{~s}$ \\
Passo de tempo OpenFOAM & $10^{-4} \mathrm{~s}$ \\
\hline
\end{tabular}

As simulações foram realizadas a fim de verificar o comportamento do coeficiente de restituição com a variação da velocidade de impacto. Para isso, as esferas foram soltas em queda livre a partir de diferentes alturas $(97,110,120,130,140$ e $150 \mathrm{~cm}$ ), conforme o procedimento experimental de Lopes et al. (2018). A partir dos dados das velocidades de impacto e de rebatimento, os coeficientes de restituição foram calculados segundo a Equação 1. As velocidades de impacto foram também empregadas para o cálculo de $e$ a partir da correlação proposta por Alsakarneh et al. (2012), conforme a Equação 2.

$$
\begin{aligned}
& e=\left|\frac{v_{\text {rebatimento }}}{v_{\text {impacto }}}\right| \\
& e=\exp ^{-\left(0,00564 . v_{\text {impacto }}+0,3033\right)}
\end{aligned}
$$

A correlação proposta por Alsakarneh et al. (2012) é derivada do modelo viscoelástico de Maxwell modificado, adaptado para esferas rígidas (módulo de Young = 6,7 x 10 ${ }^{7} \mathrm{~Pa}$ ).

\section{RESULTADOS}

\subsection{Teste de independência do número de imagens/segundo}



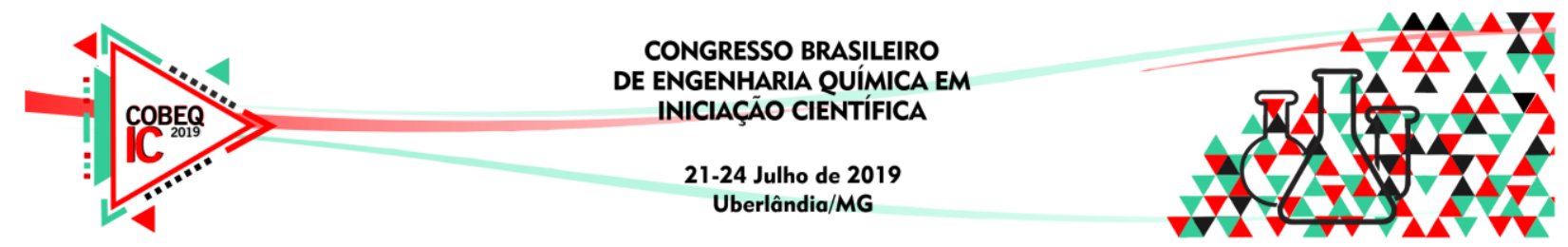

Os dados obtidos para os testes de independência do número de imagens/segundo são apresentados na Tabela 4 e, graficamente, na Figura 1, onde são comparados aos dados experimentais de Lopes et al. (2018) obtidos para altura de queda de 0,97 m.

Tabela 4 - $e$ simulado em função do número de imagens/segundo

\begin{tabular}{|c|c|c|c|c|c|}
\hline \multirow[t]{2}{*}{ Simulação } & \multirow[t]{2}{*}{ Imagens/segundo } & \multicolumn{2}{|c|}{$\begin{array}{c}\text { ABS com revestimento } \\
\text { uniforme } \\
(\boldsymbol{e} \text { experimental }=\mathbf{0 , 7 3 5})\end{array}$} & \multicolumn{2}{|c|}{$\begin{array}{c}\text { Tapioca } \\
(e \text { experimental }=0,405)\end{array}$} \\
\hline & & $e_{\text {simulado }}$ & Erro & $e_{\text {simulado }}$ & Erro \\
\hline 1 & 100 & 0,74923 & $1,936 \%$ & 0.40812 & $0.771 \%$ \\
\hline 2 & 111,11 & 0,74759 & $1,713 \%$ & 0.40722 & $0.548 \%$ \\
\hline 3 & 166,67 & 0,74271 & $1,049 \%$ & 0.42407 & $4.709 \%$ \\
\hline 4 & 200 & 0,74110 & $0,830 \%$ & 0.40918 & $1.031 \%$ \\
\hline 5 & 250 & 0,74810 & $1,783 \%$ & 0.40622 & $0.301 \%$ \\
\hline 6 & 285,71 & 0,74729 & $1,672 \%$ & 0.41386 & $2.188 \%$ \\
\hline 7 & 333,33 & 0,75080 & $2,150 \%$ & 0.40792 & $0.720 \%$ \\
\hline 8 & 400 & 0,74782 & $1,745 \%$ & 0.40876 & $0.928 \%$ \\
\hline 9 & 500 & 0,74147 & $0,818 \%$ & 0.40834 & $0.824 \%$ \\
\hline 10 & 666,67 & 0,73950 & $0,612 \%$ & 0.40536 & $0.090 \%$ \\
\hline 11 & 1000 & 0,74755 & $1,707 \%$ & 0.40714 & $0.528 \%$ \\
\hline 12 & 2000 & 0,74674 & $1,598 \%$ & 0.40791 & $0.719 \%$ \\
\hline 13 & 10000 & 0,74707 & $1,643 \%$ & 0.40846 & $0.854 \%$ \\
\hline
\end{tabular}

Figura $1-e$ em função do número de imagens/segundo para as partículas de ABS com revestimento (a) e Tapioca (b).

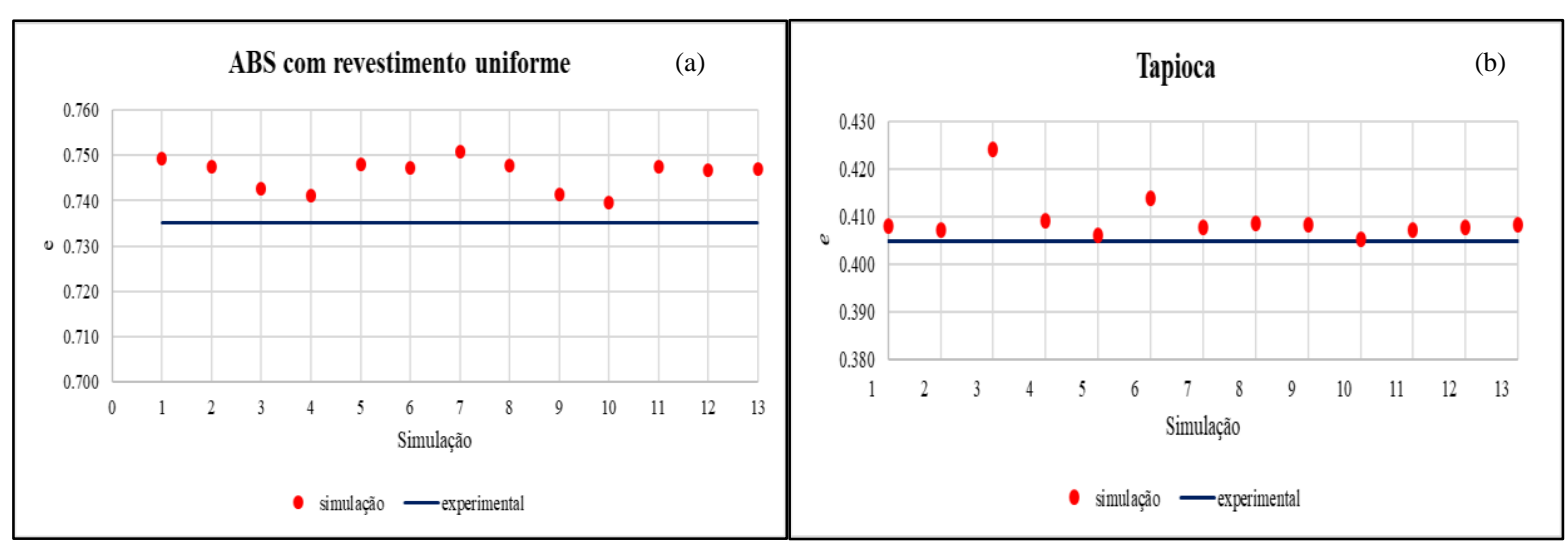

Os coeficientes de restituição variaram significativamente em relação ao experimental em faixas de maior oscilação de dados. A partir da relação 400 imagens/segundo (simulação 8), os resultados das duas partículas apresentam uma menor discrepância, além de maior proximidade entre os valores simulados e experimentais. Dessa forma, para as simulações subsequentes foi estabelecida a razão 666,67 imagens/segundo, de modo a garantir a independência dos resultados em relação ao número de passos e a proximidade com os dados experimentais.

\subsection{Influência da velocidade de impacto}

De posse dos resultados (Tabela 5), o comportamento do coeficiente de restituição em função da velocidade de impacto é ilustrado na Figura 2. 


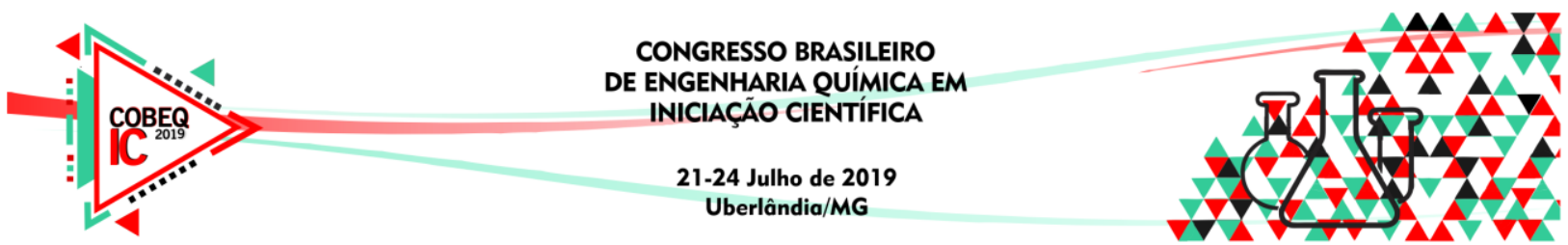

Tabela 5 - Valores de $e$ em função da velocidade de impacto

\begin{tabular}{c|ccc|ccc}
\cline { 2 - 7 } & \multicolumn{2}{|c}{ ABS com revestimento uniforme } & \multicolumn{3}{c}{ Tapioca } \\
\hline $\boldsymbol{h}$ queda $(\mathbf{m})$ & $\boldsymbol{v}$ impacto $(\mathbf{m} / \mathbf{s})$ & $\boldsymbol{e}$ simulado & $\boldsymbol{e}$ correlação & $\boldsymbol{v}$ impacto $(\mathbf{m} / \mathbf{s})$ & $\boldsymbol{e}$ simulado & $\boldsymbol{e}$ correlação \\
\hline 0,97 & 4,2778 & 0,7437 & 0,7208 & 3,8433 & 0,4024 & 0,7225 \\
1,1 & 4,5549 & 0,7364 & 0,7197 & 4,0593 & 0,4023 & 0,7217 \\
1,2 & 4,7479 & 0,7357 & 0,7189 & 4,2136 & 0,4030 & 0,7210 \\
1,3 & 4,9399 & 0,7349 & 0,7181 & 4,4633 & 0,3987 & 0,7200 \\
1,4 & 5,1177 & 0,7342 & 0,7174 & 4,6494 & 0,3975 & 0,7193 \\
1,5 & 5,2938 & 0,7290 & 0,7167 & 4,9939 & 0,3989 & 0,7179 \\
\hline
\end{tabular}

Figura 2 - Influência da velocidade colisão no coeficiente de restituição das esferas de ABS com revestimento uniforme (a) e das de Tapioca (b).

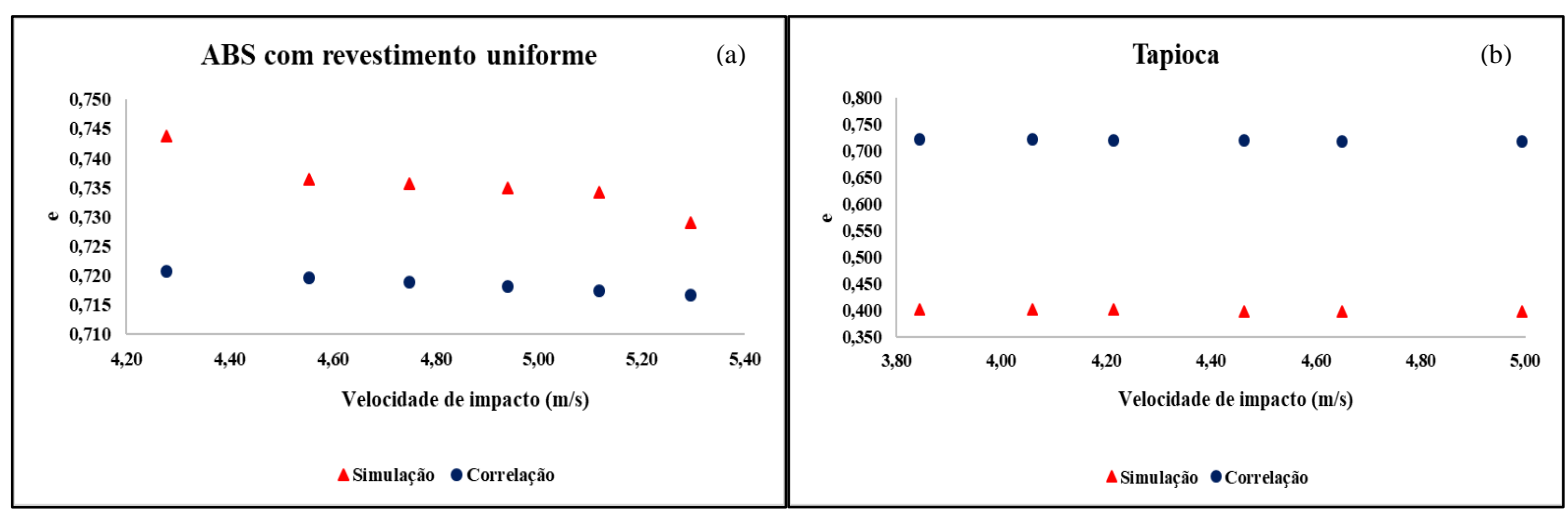

Os resultados confirmam a dependência do coeficiente de restituição com a velocidade de impacto no momento da colisão. Para as esferas de ABS observa-se uma redução progressiva nos valores desse parâmetro devido ao aumento da velocidade de colisão. Enquanto para as partículas de tapioca, os coeficientes de restituição oscilam em torno de valores próximos, indicando menor influência da velocidade de impacto para essas partículas. Além disso, a variação de e é também efeito da elasticidade do material. As esferas de ABS, com maior módulo de Young, apresentaram elevados coeficientes, enquanto valores significativamente menores foram obtidos para as de tapioca.

O módulo de Young proporciona uma medida de rigidez e está associado à resistência à deformação do material. Para as partículas mais rígidas, nas colisões em ar, a maior parte da energia armazenada é convertida em energia cinética, sem que ocorram grandes dissipações de energia na forma de calor, deformação ou atrito. Portanto, possuem maior velocidade de rebatimento e, consequentemente, maior coeficiente de restitução. Enquanto as partículas macias sofrem maior deformação e dissipam grande parte da energia após a colisão.

Comparando os dados de $e_{\text {simulado }}$ e $e_{\text {correlação }}$ para as esferas de ABS, observam-se baixos desvios (entre 1,6\% e 3\%), o que indica que a correlação proposta por Alsakarneh et al. (2012) foi capaz de prever de forma satisfatória o coeficiente de restituição dessas partículas. No entanto, desvios superiores a $70 \%$ para as esferas de tapioca demonstram que o modelo teórico não é adequado para descrever o coeficiente de restituição de partículas macias. A correlação considera que todo o corpo retorna a suas dimensões originais após a colisão e, portanto, despreza as deformações e grandes dissipações de energia no sistema, o que se comprova pelos valores obtidos. 


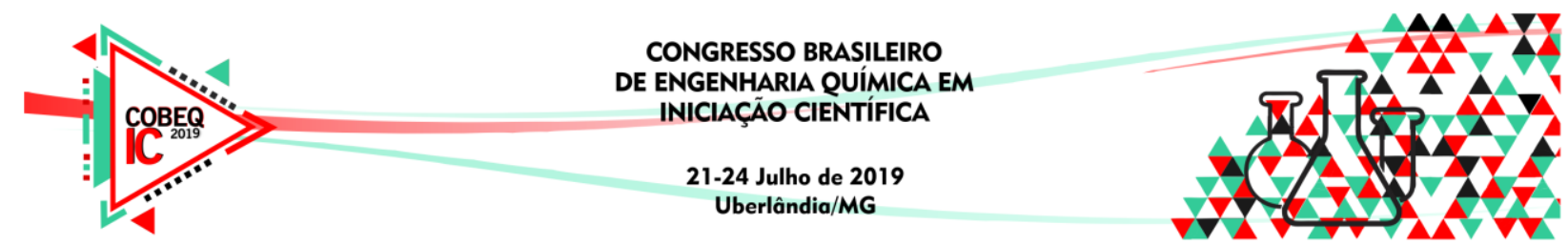

\section{CONCLUSÕES}

Os coeficientes de restituição simulados para altura de $0,97 \mathrm{~m}$ mostraram-se próximos aos experimentais e confirmaram a influência das propriedades elásticas do material, com maior coeficiente de restituição para partículas de ABS, as quais possuem maior módulo de Young e dissipam menos energia após a colisão.

Os resultados indicam que as simulações conseguiram prever de forma satisfatória o comportamento desse parâmetro, demonstrando a influência da velocidade de impacto sobre o coeficiente de restituição. O que se observa principalmente para as esferas de ABS, que apresentou uma redução progressiva desse parâmetro para maiores velocidades de impacto. Para as partículas de tapioca notaram-se valores bastante aproximados, indicando uma menor influência da velocidade de impacto.

A correlação proposta por Alsakarneh et al. (2012) forneceu resultados satisfatórios para as esferas de ABS, mostrando-se adequada para descrever o comportamento de colisões de partículas rígidas, com maior módulo de Young.

\section{AGRADECIMENTOS}

O presente trabalho foi realizado com apoio da Coordenação de Aperfeiçoamento de Pessoal de Nível Superior - Brasil (CAPES) - Código de Financiamento 001, da Fundação de Amparo à Pesquisa do Estado de São Paulo (FAPESP) - Projeto 2018/20711-2, e do Conselho Nacional de Desenvolvimento Científico e Tecnológico (CNPq).

\section{REFERÊNCIAS}

ALBUQUERQUE, M. V. Modelagem e Análise Dinâmica de um Absorvedor de Vibrações por Efeito de Impacto. Dissertação de mestrado em Engenharia Mecânica, Universidade Estadual de Campinas - Unicamp. Campinas/SP, 2016.

ALSAKARNEH, A.; QUINN, B.; KELLY, G.; BARRETT, J. Modeling and simulation of the coefficient of restitution of the sliotar in hurling. Sport. Biomech. v. 11, p. 342-357, 2012 .

DU, W.; BAO, X.; XU, J.; WEI, W. Computational fluid dynamics (CFD) modeling of spouted bed: Influence of frictional stress, maximum packing limit and coefficient of restitution of particles. Chem. Eng. Sci. v. 61, p. 4558-4570, 2006.

GONDRET, P.; LANCE, M.; PETIT, L. Bouncing motion of spherical particles in fluids. Phys. Fluids. v.14(2), p. 643-652, 2002.

JOSEPH, G; ZENIT, R.; HUNT, M; ROSENWINKEL, A. Particle-wall collisions in a viscous fluid. J. Fluid Mech., v. 433, p. 329-346, 2001.

LOPES, G. C.; BI, X; EPSTEIN, N.; BALDWIN, S.; GRACE, J. R. Hydrodynamic characteristics of particles with different roughness and deformability in a liquid fluidized bed. Chem. Eng. Sci., v. 185, p. 50-63, 2018. 